dependence. By being clear and direct the guidelines provide at least a basis to start on. One hopes that Dr MacDonald's letter will prompt more of us to dig out the bright orange A4 size booklet, which having arrived in November is now in danger of being put aside, and take a fresh look at it.

TOM WALKER

London N1 OEG

ARTHUR BANKS

Chelmsford CM2 ODB

1 Jollife DW, Melville AW"I. Treatment of heroin addiction. $\mathcal{F} R$ Coll Gen Pract 1983;33:368.

Withdrawal symptoms and rebound anxiety after six week course of diazepam

SIR,-Mr K G Power and his colleagues (27 April, p 1246) gave diazepam to 10 patients for six weeks and made comparisons with 11 patients who received placebos. The authors claim to have found rebound anxiety and other withdrawal symptoms after the diazepam. Their evidence is unconvincing.

A word means only what it is usually taken to mean, but in Europe and the USA to claim a "rebound" effect would require that after stopping diazepam the patients should be more anxious than they would have been had they never taken the drug - that is, worse than baseline. This was not so in the case of the patients of Mr Power and others. When the drug stopped their patients merely became about as anxious as before.

When, alternatively, the authors considered the placebo group and the diazepam group in relation to each other and in relation to time, using analyses of variance no significant effect of the drug and no significant interaction of drug with time emerged. And that really should be that.

If one looks at the degrees of freedom in their repeated measures analysis of variance in each case only a single dimension is given, and one wonders where has the necessary second dimension gone. I read the text with care but remain baffled by table II. What I do take from it is that arithmetic addition has been used for symptoms like anxiety and apprehension, thereby surely counting the same thing twice-a suspect practice.

All this does not mean there cannot be measurable and genuine rebound anxiety, ${ }^{1}$ insomnia, ${ }^{2}$ or raised cortisol concentrations ${ }^{3}$ after intake of benzodiazepines, and after shorter periods than six weeks, but I cannot conclude that the paper in your journal has shown what is claimed.

IAN OSWALD

University Department of Psychiatry,

Royal Edinburgh Hospital,

Royal Edinburgh Hosp

1 Allen S, Oswald I. Anxiety and sleep after forazepam. $\mathrm{Br} \mathcal{J}$ Clin Pharmacol 1976;3:165-8.

2 Oswald I, French C, Adam K, Gilham J. Benzodiazepine hypnotics remain effective for 24 weeks. Br Med $\mathcal{F}$ 1982;284 860-2.

3 Adam K, Oswald I, Shapiro C. Effects of loprazolam and triazolam on sleep and overnight urinary cortisol. Psychopharmacology 1984;82:389-94.

${ }_{*}^{*} \mathrm{Mr}$ Power replies below.-ED, $B M \mathcal{F}$.

SIR-Professor Oswald rightly states that the term "rebound" has come to denote a specific worsening of symptoms in comparison to baseline. Perhaps the title of our paper would have been more appropriate if we had used the term "recurrence" rather than "rebound." However, our finding was that whereas both diazepam and placebo groups showed no significant differences in mean scores at baseline, the diazepam group showed significantly higher Hamilton anxiety scale ratings at the end of withdrawal compared with the end of active treatment whereas the placebo group showed no such change.

Secondly, Professor Oswald states that we found no significant effect of the drug, nor a significant interaction of drug with time, using an analysis of variance. This is incorrect (as stated in the article "a significant drug $\times$ time of assessment interaction effect $(\mathrm{F}=3.67 ; \mathrm{df}=4 ; \mathrm{p}<0.009)$ " was found.)

Thirdly, Professor Oswald rightly notes that in each case only a single degree of freedom was quoted. However, the second degree of freedom dimension-namely, the degree of freedom for error - can be easily calculated from the data in the text and makes no positive or negative contribution to the significance of the results.

Fourthly, Professor Oswald says that he is "baffled" by our table II, on adverse withdrawal reactions. The table shows the emergence of an increase in previously occurring symptoms or the emergence of new symptoms. No patient may have a score in both columns for any one symptom. This method is derived from the definition of Owen and Tyrer of the necessary effects to show a withdrawal syndrome. ${ }^{1}$ Although Professor Oswald states that there is an overlap of categories in table II we would contend that the only overlap that occurred was between the first general category of "anxiety" and any subsequent more specific symptoms of withdrawal.

In conclusion, we would accept that a narrow definition of "rebound" has not been met, but all the other claims in the paper are wholly justified and we have shown a sufficiently clear withdrawal effect to merit further serious study. We shall be undertaking further studies to elucidate whether the effect is seen in graded withdrawal.

K G Power

Department of Psychology,

University of Stirling,

Stirling FK9 4LA

1 Owen RT, Tyrer P. Benzodiazepine dependence. A review of the evidence. Drugs 1983;25,385-98.

\section{Relation between cancer of the colon and} blood transfusion

SIR,-We were interested to read the report of Professor Neil Blumberg and colleagues (6 April, p 1037) on an association between perioperative blood transfusion and colorectal cancer recurrence. We have also investigated this relationship and our results are at variance with those reported by them and by Burrows and Tartter. ${ }^{1}$

A prospective study was undertaken between 1 March 1981 and 28 February 1982 of all new cases of colorectal cancer in Auckland, New Zealand. Of 288 patients presenting to one of three regional public hospitals, 179 underwent potentially curative surgery. Data on transfusion were available for $174(97 \%)$ patients. Information on tumour site, differentiation, and Dukes's classification was obtained from a computer held database. All patients have been followed up at six monthly intervals for at least three years. The $\chi^{2}$ analysis was used for evaluating the statistical significance between proportions.

One hundred and three patients received transfusions and 71 did not. There was no significant difference between the two groups in terms of patient age, tumour differentiation, or Dukes's stage. The proportion of patients with Dukes's C tumours was $34 / 103(33 \%)$ in the transfused group and $24 / 71(34 \%)$ in the non-transfused group. There were significantly more rectal tumours in those who received transfusions $(\mathrm{p}<0.05)$ and in patients undergoing operations longer than two and a half hours $(\mathrm{p}<0.001)$. Recurrence occurred in 42 patients $(24 \%)$, and 33 have died from it.
Recurrence developed in $17 / 71$ (24\%) patients in the non-transfused group and $25 / 103(24 \%)$ in the transfused group. The cumulative percentage of patients surviving disease free in the two groups can be seen in the table. There was no statistical difference between the distributions of time to recurrence using the log rank test $\left(\chi^{2}=0.29\right.$ on $\left.1 \mathrm{df}, \mathrm{p}>0.59\right)$.

Cumulative percentage surviving free of recurrence

Time from operation

(months): $\begin{array}{lllllll}6 & 12 & 18 & 24 & 30 & 36 & 42\end{array}$

$\begin{array}{lllllllll}\text { Transfused patients } & 96 & 88 & 83.8 & 77 \cdot 2 & 76 & 73.6 & 73.6\end{array}$ $\begin{array}{llllllll}\text { Non-transfused patients } & 95 \cdot 7 & 88 \cdot 4 & 88 \cdot 4 & 86 \cdot 9 & 79 \cdot 4 & 77 \cdot 8 & 70 \cdot 4\end{array}$

While a significant association between Dukes's staging and recurrence was observed $(p<0 \cdot 001)$, we have been unable to confirm an association between perioperative blood transfusion and recurrence of colorectal cancer within three years of a prospective study. Until this relation is further clarified we do not believe that firm recommendations about the deliberate withholding of blood transfusion from patients undergoing colorectal cancer surgery can be made. However, we agree with Burrows and Tartter that transfusion should be avoided unless absolutely necessary. In this study $25 \%$ of the patients in the transfused group received two units or less, and it seems likely that transfusion with its attendant hazards could be avoided in a significant number of patients.

P D FRANKISH

Gartnavel General Hospital,

Glasgow GIZ 0YN

RAEWYN K MCNEE

Department of Surgery,

Auckland University School of Medicine,

Auckland, New Zealand

P G Alley

North Shore Hospital,

Auckland, New Zealand

D G WOODFIELD

Blood Transfusion Service,

Auckland Hospital,

Auckland, New Zealand

1 Burrows L, Tartter P. Effect of blood transfusions on colonic malignancy recurrence rate. Lancet 1982;ii:662.

\section{Rubella: immunity and vaccination in} schoolgirls

SIR-We are surprised to read of the proposal by Dr Goura Kudesia and her collegues in Glasgow (11 May, p 1406) that a larger scale study is needed to provide a clearer understanding of the organisational and technical problems encountered in implementing a programme of serological screening of 11-13 year old girls to identify those who already have natural immunity to rubella and those who require vaccine.

Exactly such a programme, which has been operational in Edinburgh since 1970, has already been reported ${ }^{12}$ and referred to in your columns. Since 1970 complete cohorts of about 3500 girls a year in Edinburgh have been offered serological screening of capillary blood to test their immunity to rubella. Samples are collected straight into previously numbered precipitin tubes, which are delivered to the laboratory the same day. Girls who are found to have no rubella antibodies are immunised and retested to ensure seroconversion. The acceptance rate for this programme has been consistently good $(97 \%)$ and the mean proportion of girls requiring vaccine has been $27 \%$ of 55696 tested over 15 years. All results are reported to the individual girl and to her practitioner. 